\title{
Advances in the characterization of non-conventional resources with potential use in animal nutrition
}

\author{
M. Rosales M., T. R. Preston, J. E. Vargas \\ Convenio Interinstitucional para la Producción Pecuaria en el Valle del Rio Cauca (CIPAV), Apartado Aéreo 7482, \\ Cali, Columbia
}

\section{Introduction}

The tropical zones hold the biggest genetic diversity in the whole world, diversity which is expressed in the large number of vascular plants per unit of surface. Non-conventional forage plants are an important example of this huge natural potential. However, several of these plants have developed defence mechanisms to protect themselves against predators; these mechanisms are mainly: phenols, alkaloids, saponins and steroids. Because of this the characterization of these natural resources must be a research priority. Most of these plants are unknown to researchers, but not to peasants who have collected a lot of knowledge (communicated from generation to generation) about the use of most of these resources.

\section{Methods}

The aim of this characterization is to identify a group of forages which could be used in different tropical regions. Most of the observations about use have been obtained from peasants and farmers; the rest are quite well known forage plants. Table 1 presents the updated list of the plants characterized by CIPAV.

Table 1 Updated list of the plants characterized by CIPAV

\begin{tabular}{|c|c|c|c|c|c|}
\hline Scientific name & $\begin{array}{l}\text { Common } \\
\text { name }\end{array}$ & Family & $\begin{array}{l}\text { Kind of } \\
\text { plant }\end{array}$ & $\begin{array}{l}\text { Part of } \\
\text { plant }\end{array}$ & $\begin{array}{l}\text { Potential use } \\
\text { (animal species) }\end{array}$ \\
\hline Gliricidia sepium & Matarratón & $\begin{array}{l}\text { Leguminosae/ } \\
\text { Papilionacea }\end{array}$ & Tree & Leaves & Ruminants \\
\hline Leucaena leucocephala & Leucaena & $\begin{array}{l}\text { Leguminosae/ } \\
\text { Mimosacea }\end{array}$ & Tree & Leaves & Ruminants \\
\hline Trichantera gigantea & Nacedero & Acantacea & Tree & Leaves & $\begin{array}{l}\text { Monogastrics, } \\
\text { ruminants }\end{array}$ \\
\hline Inga spectabilis & Guamo & $\begin{array}{l}\text { Leguminosae/ } \\
\text { Mimosacea }\end{array}$ & Tree & Leaves & Toxic plant \\
\hline Canavalia ensiformis & Canavalia & $\begin{array}{l}\text { Leguminosae/ } \\
\text { Papilionacea }\end{array}$ & Shrub & Fruits & $\begin{array}{l}\text { Poultry (maximum } 30 \% \\
\text { in diet). Toxic for pigs }\end{array}$ \\
\hline Cajanus cajan & Guandul & $\begin{array}{r}\text { Leguminosae/ } \\
\text { Papilionacea }\end{array}$ & Shrub & Leaves & Ruminants \\
\hline Hibiscus rosasinencis & San joaquin & Malvacea & Shrub & Leaves & Pigs, ruminants \\
\hline Urera caracasama & Pringamoza & Urticacea & Shrub & Leaves & Monogastrics, ruminants \\
\hline Enterolobium ciclocarpum & Orejero & $\begin{array}{l}\text { Leguminosae/ } \\
\text { Mimosacea }\end{array}$ & Tree & Leaves & $\begin{array}{l}\text { Ruminants, reduce population } \\
\text { of rumen protozoa }\end{array}$ \\
\hline Simphytum peregrinum & Comfrey & & Herbaceous & Leaves & $\begin{array}{l}\text { Monogastrics, rabbits, } \\
\text { guinea pigs }\end{array}$ \\
\hline $\begin{array}{l}\text { Azolla pinnata } \\
\text { Erythrina poeppigiana }\end{array}$ & $\begin{array}{l}\text { Azolla } \\
\text { Cachimbo }\end{array}$ & $\begin{array}{l}\text { Azollacea } \\
\text { Leguminosae/ } \\
\text { Papilonacea }\end{array}$ & $\begin{array}{l}\text { Aquatic } \\
\text { Tree }\end{array}$ & $\begin{array}{l}\text { Leaves } \\
\text { Leaves }\end{array}$ & $\begin{array}{l}\text { Ruminants, monogastrics } \\
\text { Ruminants }\end{array}$ \\
\hline
\end{tabular}




\section{Results}

The results of chemical analysis of these foods are summarized in Table 2.

All but one of the forages presented in Table 2 are being used as food resources in different animal species, and their anti-nutritional factors are a matter of research. The Inga spectabilis has been shown to be highly toxic (cardiac depressive effect).
Other potential resources, which have been used by peasants and farmers, are being evaluated at the moment, and are shown in Table 3 .

\section{Acknowledgements}

M. Rosales acknowledges IFS grant no. B/1441-1 and J. E. Vargas acknowledges IFS grant no. B/1798-1.

Table 2 Chemical analysis of forages

\begin{tabular}{|c|c|c|c|c|c|c|c|c|c|}
\hline Scientific name & $\begin{array}{c}\text { Dry } \\
\text { matter } \\
(\mathrm{DM})(\mathrm{g} / \mathrm{kg})\end{array}$ & $\begin{array}{l}\text { Nitrogen } \\
(g / k g \text { DM) }\end{array}$ & $\begin{array}{c}\text { Protein } \\
\text { (g/kg DM) }\end{array}$ & $\begin{array}{c}\text { Calcium } \\
(\mathrm{g} / \mathrm{kg})\end{array}$ & $\begin{array}{c}\text { Phosphorus } \\
\text { (g/kg) }\end{array}$ & $\begin{array}{c}\text { Potassium } \\
(\mathrm{g} / \mathrm{kg})\end{array}$ & $\begin{array}{c}\text { Magnesium } \\
(\mathbf{g} / \mathbf{k g})\end{array}$ & $\begin{array}{l}\text { Degradability } \\
\text { (proportion } \\
\text { per 24 h) }\end{array}$ & $\begin{array}{l}\text { mg total } \\
\text { phenols per } \\
\text { of plant (DM) }\end{array}$ \\
\hline Gliricidia sepium & 208.7 & 40.5 & $253 \cdot 1$ & $13 \cdot 2$ & 3.0 & 214 & 50 & 0.72 & 10.923 \\
\hline Leucaena leucocephala & 2500 & 39.1 & $244 \cdot 3$ & 19.8 & 160 & 110 & $4 \cdot 2$ & 061 & $42 \cdot 328$ \\
\hline Trichantera giganten & 2246 & $27 \cdot 1$ & $169 \cdot 3$ & $24 \cdot 0$ & 3.8 & $24-2$ & 90 & 0.60 & 50.288 \\
\hline Inga spectabilis & 5500 & 26.7 & $166 \cdot 9$ & 1.5 & 0.7 & 49 & 14 & 0.30 & 18.947 \\
\hline Canavalia ensiformis & 278.9 & 41.7 & 260.7 & 156 & 0.4 & 280 & $4 \cdot 3$ & 0.77 & 14.720 \\
\hline Cajamus cajan & 260.5 & 42.7 & 2668 & 10.5 & 3.9 & 21.7 & 3.1 & 0.56 & 12.944 \\
\hline Hibiscus rosasinencis & 2100 & $33 \cdot 3$ & 208.1 & 10.5 & 46 & 284 & $5 \cdot 5$ & 0.83 & 12.903 \\
\hline Urera caracasama & 179.0 & 25.9 & 161.8 & 19.1 & 6.3 & 39.0 & 6.8 & 0.72 & 9.580 \\
\hline Enterolobium ciclocarpum & 300.0 & 23.0 & 143.7 & $14 \cdot 1$ & 1.1 & 8.8 & 10.7 & 0.35 & 13.787 \\
\hline Simphytum peregrinum & 118.9 & 32.1 & 2006 & $15-0$ & 7.8 & 664 & 4.7 & 0.75 & 9.985 \\
\hline Azolla pinnata & 7000 & 35.9 & 2243 & 11.7 & 3.3 & 21.2 & $5-9$ & 0.47 & 8.790 \\
\hline Erythrina poeppigiana & 232.3 & $38 \cdot 3$ & 239.3 & $16 \cdot 8$ & 3.4 & 16.8 & $4 \cdot 3$ & 0.54 & 11.931 \\
\hline
\end{tabular}

Table 3 Other potential foods

\begin{tabular}{|c|c|c|c|c|c|}
\hline Scientific name & $\begin{array}{l}\text { Common } \\
\text { name }\end{array}$ & Family & $\begin{array}{l}\text { Kind of } \\
\text { plant }\end{array}$ & $\begin{array}{l}\text { Part of } \\
\text { plant }\end{array}$ & $\begin{array}{c}\text { Potential use } \\
\text { (animal species) }\end{array}$ \\
\hline Eichorna crassipes & Buchón & Pontederiacea & Aquatic & Whole plant & Ruminants \\
\hline Helodea spp. & Elodea & Hidrocaritacea & Aquatic & Stem & Ruminants \\
\hline Spirogira spp. & Spirogira & & Aquatic & Whole plant & Ruminants \\
\hline Amaranthus dubius & Bledo & Amaranthacea & Herbaceous & Whole plant & Monogastrics \\
\hline Morus alba & Morera & Moracea & Herbaceous & Whole plant & Monogastrics \\
\hline Bidens pilosa & Papunga & Compositae & Herbaceous & Leaves & Monogastrics, rabbits \\
\hline Renealmia occidentael & Sanjuanito & Zingiberacea & Herbaceous & Whole plant & Ruminants \\
\hline Thitonia diversifolia & Botón de oro & Compositacea & Shrub & Whole plant & Monogastrics \\
\hline Phyllanthus acuminatus & Chirrinchao & Euphorbiacea & Shrub & Leaves & Monogastrics \\
\hline Ricinus communis & Higuerilla & Euphorbiacea & Shrub & Leaves & Ruminants \\
\hline Erythrina edulis & Chachafruto & $\begin{array}{l}\text { Leguminosae/ } \\
\text { Papilionacea }\end{array}$ & Tree & Leaves & $\begin{array}{l}\text { Ruminants (leaves), } \\
\text { monogastrics (fruits) }\end{array}$ \\
\hline Erythrina glauca & Pízamo & $\begin{array}{l}\text { Leguminosae/ } \\
\text { Papilionacea }\end{array}$ & Tree & Leaves & Ruminants \\
\hline Mangifera indica & Mango & Anacardinacea & Tree & Leaves & $\begin{array}{l}\text { Ruminants (leaves), } \\
\text { monogastrics (fruits) }\end{array}$ \\
\hline Guazuma ulmifolia & Guásimo & UImacea & Tree & Leaves & Ruminants \\
\hline
\end{tabular}

